\title{
On Planar Greedy Drawings of 3-Connected Planar Graphs*
}

\author{
Giordano Da Lozzo', Anthony D'Angelo², and Fabrizio Frati ${ }^{3}$ \\ 1 Department of Computer Science, University of California, Irvine, CA, USA \\ gdalozzo@uci.edu \\ 2 School of Computer Science, Carleton University, Ottawa, Canada \\ anthonydangelo@cmail. carleton.ca \\ 3 Department of Engineering, Roma Tre University, Rome, Italy \\ frati@dia.uniroma3.it
}

\begin{abstract}
A graph drawing is greedy if, for every ordered pair of vertices $(x, y)$, there is a path from $x$ to $y$ such that the Euclidean distance to $y$ decreases monotonically at every vertex of the path. Greedy drawings support a simple geometric routing scheme, in which any node that has to send a packet to a destination "greedily" forwards the packet to any neighbor that is closer to the destination than itself, according to the Euclidean distance in the drawing. In a greedy drawing such a neighbor always exists and hence this routing scheme is guaranteed to succeed.

In 2004 Papadimitriou and Ratajczak stated two conjectures related to greedy drawings. The greedy embedding conjecture states that every 3-connected planar graph admits a greedy drawing. The convex greedy embedding conjecture asserts that every 3-connected planar graph admits a planar greedy drawing in which the faces are delimited by convex polygons. In 2008 the greedy embedding conjecture was settled in the positive by Leighton and Moitra.

In this paper we prove that every 3-connected planar graph admits a planar greedy drawing. Apart from being a strengthening of Leighton and Moitra's result, this theorem constitutes a natural intermediate step towards a proof of the convex greedy embedding conjecture.
\end{abstract}

1998 ACM Subject Classification G.2.2 Graph Theory

Keywords and phrases Greedy drawings, 3-connectivity, planar graphs, convex drawings

Digital Object Identifier 10.4230/LIPIcs.SoCG.2017.33

\section{Introduction}

Geographic routing is a family of routing protocols for ad-hoc networks, which are networks with no fixed infrastructure - such as routers or access points - and with dynamic topology [15, $27,28]$. In a geographic routing scheme each node of the network actively sends, forwards, and receives packets; further, it does so by only relying on the knowledge of its own geographic coordinates, of those of its neighbors, and of those of the packet destination. Greedy routing is the simplest and most renowned geographic routing scheme. In this protocol, a node that has to send a packet simply forwards it to any neighbor that is closer - according to the Euclidean distance - to the destination than itself. The greedy routing scheme might fail

\footnotetext{
* This article reports on work supported by the U.S. Defense Advanced Research Projects Agency (DARPA) under agreement no. AFRL FA8750-15-2-0092. The views expressed are those of the authors and do not reflect the official policy or position of the Department of Defense or the U.S. Government. This research was also partially supported by NSERC, by MIUR-PRIN Project 20157EFM5C - "MODE", and by H2020-MSCA-RISE Project 734922 - "CONNECT".
}

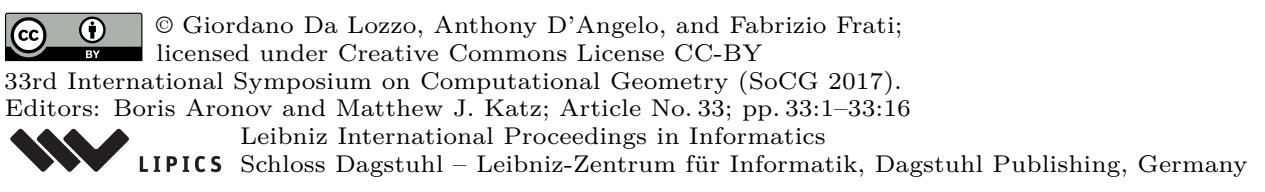


to deliver packets because of the presence of a void in the network; this is a node with no neighbor closer to the destination than itself. For this reason, several variations of the greedy routing scheme have been proposed; see, e.g., [6, 19, 20].

Apart from its failure in the presence of voids, the greedy routing protocol has two disadvantages which limit its applicability. First, in order for the protocol to work, each node of the network has to be equipped with a GPS, which might be expensive and might consume excessive energy. Second, two nodes that are close geographically might be unable to communicate with each other because of the presence of topological obstructions. Rao et al. [26] introduced the following brilliant idea for extending the applicability of geographic routing in order to overcome the above issues. Suppose that a network topology is known; then one can assign virtual coordinates to the nodes and use these coordinates instead of the geographic locations of the nodes in the greedy routing protocol. The virtual coordinates can then be chosen so that the greedy routing protocol is guaranteed to succeed.

Assigning the virtual coordinate to the nodes of a network corresponds to the following graph drawing problem: given a graph $G$, construct a greedy drawing of $G$, that is a drawing in the plane such that, for any ordered pair of vertices $(x, y)$, there is a neighbor of $x$ in $G$ that is closer - in terms of Euclidean distance - to $y$ than $x$. Equivalently, a greedy drawing of $G$ is such that, for any ordered pair of vertices $(x, y)$, there is a distance-decreasing path from $x$ to $y$, that is, a path $\left(u_{1}, \ldots, u_{m}\right)$ in $G$ such that $x=u_{1}, y=u_{m}$, and the Euclidean distance between $u_{i+1}$ and $u_{m}$ is smaller than the one between $u_{i}$ and $u_{m}$, for any $i=1, \ldots, m-2$.

Greedy drawings experienced a dramatical surge of popularity in the theory community in 2004, when Papadimitriou and Ratajczak [24] proposed the following two conjectures about greedy drawings of 3-connected planar graphs (the convex greedy embedding conjecture has not been stated in the journal version [25] of their paper [24]).

- Conjecture 1 (Greedy embedding conjecture). Every 3-connected planar graph admits a greedy drawing.

- Conjecture 2 (Convex greedy embedding conjecture). Every 3-connected planar graph admits a convex greedy drawing.

Papadimitriou and Ratajczak [24, 25] provided several reasons why 3-connected planar graphs are central to the study of greedy drawings. First, there exist non-3-connected planar graphs and 3-connected non-planar graphs that do not admit any greedy drawing. Thus, the 3-connected planar graphs form the largest class of graphs that might admit a greedy drawing, in a sense. Second, all the 3 -connected graphs with no $K_{3,3}$-minor admit a 3 -connected planar spanning graph, hence they admit a greedy drawing, provided the truth of the greedy embedding conjecture. Third, the preliminary study of Papadimitriou and Ratajczak [24, 25] provided evidence for the mathematical depth of their conjectures.

Leighton and Moitra [21] (and, independently and slightly later, Angelini et al. [4]) settled Conjecture 1 in the affirmative. In this paper we show the following result.

- Theorem 3. Every 3-connected planar graph admits a planar greedy drawing.

Given a 3 -connected planar graph $G$, the algorithms in [4, 21] find a spanning subgraph $S$ of $G$ and construct a (planar) greedy drawing of $S$; then they embed the edges of $G$ not in $S$ as straight-line segments obtaining a, in general, non-planar greedy drawing of $G$. Thus, Theorem 3 strengthens Leighton and Moitra's and Angelini et al.'s results. Furthermore, convex drawings, in which all the faces are delimited by convex polygons, are planar, hence Theorem 3 provides a natural step towards a proof of Conjecture 2. 
Our proof employs a structural decomposition for 3-connected planar graphs which finds its origins in a paper by Chen and $\mathrm{Yu}$ [7]. This decomposition actually works for a super-class of the 3-connected planar graphs known as strong circuit graphs. We construct a planar greedy drawing of a given strong circuit graph $G$ recursively: we apply the structural decomposition to $G$ in order to obtain some smaller strong circuit graphs, we recursively construct planar greedy drawings for them, and then we suitably arrange these drawings together to get a planar greedy drawing of $G$. For this arrangement to be feasible, we need to ensure that the drawings we construct satisfy some restrictive geometric requirements; these are described in the main technical theorem of the paper - Theorem 8.

Related results. Planar greedy drawings always exist for maximal planar graphs [11]. Further, every planar graph $G$ with a Hamiltonian path $P=\left(u_{1}, \ldots, u_{n}\right)$ has a planar greedy drawing. Namely, construct a planar straight-line drawing $\Gamma$ of $G$ such that $y\left(u_{1}\right)<\cdots<$ $y\left(u_{n}\right)$; such a drawing always exists [12]; scale $\Gamma$ down horizontally, so that $P$ is "almost vertical". Then, for any $1 \leq i<j \leq n$, the paths $\left(u_{i}, u_{i+1} \ldots, u_{j}\right)$ and $\left(u_{j}, u_{j-1} \ldots, u_{i}\right)$ are distance-decreasing. A characterization of the trees that admit a (planar) greedy drawing is known [22]; indeed, a greedy drawing of a tree is always planar [2].

Algorithms have been designed to construct succinct greedy drawings, in which the vertex coordinates are represented with a polylogarithmic number of bits $[13,16,17]$; this has been achieved by allowing the embedding space to be different from the Euclidean plane or the metric to be different from the Euclidean distance.

Planar drawings have been studied in which paths between pairs of vertices are required to exist satisfying properties other than being distance-decreasing. We say that a path $P=\left(u_{1}, \ldots, u_{m}\right)$ in a graph drawing is self-approaching $[1,23]$ if, for any three points $a, b, c$ in this order along $P$ from $u_{1}$ to $u_{m}$, the Euclidean distance between $a$ and $c$ is larger than the one between $b$ and $c$-then a self-approaching path is also distance-decreasing. We say that $P$ is increasing-chord $[1,10,23]$ if it is self-approaching in both directions. We say that $P$ is strongly monotone $[3,14,18]$ if the orthogonal projections of the vertices of $P$ on the line $\ell$ through $u_{1}$ and $u_{m}$ appear in the order $u_{1}, \ldots, u_{m}$. It has been recently proved [14] that every 3-connected planar graph has a planar drawing in which every pair of vertices is connected by a strongly monotone path.

Because of space limitations some proofs are sketched or omitted; they can be found in the complete version of the paper [8].

\section{Preliminaries}

In this section we introduce some preliminaries.

Subgraphs and connectivity. We denote by $V(G)$ and $E(G)$ the vertex and edge sets of a graph $G$, respectively. For $U \subseteq V(G)$, we denote by $G-U$ the graph obtained from $G$ by removing the vertices in $U$ and their incident edges. Further, if $e \in E(G)$, we denote by $G-e$ the graph obtained from $G$ by removing the edge $e$. Let $H$ be a subgraph of $G$. An $H$-bridge $B$ of $G$ is either an edge of $G$ not in $H$ with both the end-vertices in $H$ (then $B$ is a trivial $H$-bridge), or a connected component of $G-V(H)$ together with the edges from that component to the vertices in $V(H)$ (then $B$ is a non-trivial $H$-bridge); the vertices in $V(H) \cap V(B)$ are the attachments of $B$ in $H$. For a vertex $v \in V(G)-V(H)$, we denote by $H \cup\{v\}$ the subgraph of $G$ composed of $H$ and of the isolated vertex $v$. 
A vertex $k$-cut (in the following simply called $k$-cut) in a connected graph $G$ is a set of $k$ vertices whose removal disconnects $G$. For $k \geq 2$, a connected graph is $k$-connected if it has no $(k-1)$-cut. A $k$-connected component of a graph $G$ is a maximal $k$-connected subgraph of $G$. Given a 2-cut $\{a, b\}$ in a 2-connected graph $G$, an $\{a, b\}$-component is either the edge $a b$ (then the $\{a, b\}$-component is trivial) or a subgraph of $G$ induced by $a, b$, and the vertices of a connected component of $G-\{a, b\}$ (then the $\{a, b\}$-component is non-trivial).

Plane graphs and embeddings. A drawing of a graph is planar if no two edges cross. A plane graph is a planar graph with a plane embedding; a plane embedding of a connected planar graph $G$ is an equivalence class of planar drawings of $G$, where two drawings $\Gamma_{1}$ and $\Gamma_{2}$ are equivalent if: (i) the clockwise order of the edges incident to each vertex $v \in V(G)$ coincides in $\Gamma_{1}$ and $\Gamma_{2}$; and (ii) the clockwise order of the edges of the walks delimiting the outer faces of $\Gamma_{1}$ and $\Gamma_{2}$ is the same. When we talk about a planar drawing of a plane graph $G$, we always mean that it respects the plane embedding of $G$. We assume that any subgraph $H$ of $G$ is associated with the plane embedding obtained from the one of $G$ by deleting the vertices and edges not in $H$. A vertex of $G$ is external or internal depending on whether it is or it is not incident to the outer face of $G$, respectively. For two external vertices $u$ and $v$ of a 2-connected plane graph $G$, let $\tau_{u v}(G)$ and $\beta_{u v}(G)$ be the paths composed of the vertices and edges encountered when walking along the boundary of the outer face of $G$ in clockwise and counter-clockwise direction from $u$ to $v$, respectively. Note that $\tau_{u v}(G)$ and $\beta_{v u}(G)$ have the same vertices and edges, however in reverse linear orders.

Geometry. In this paper every angle is measured in radians. The slope of a half-line $\ell$ is defined as follows. Denote by $p$ the starting point of $\ell$ and let $\ell^{\prime}$ be the vertical half-line starting at $p$ and directed towards decreasing $y$-coordinates. Then the slope of $\ell$ is the angle spanned by a counter-clockwise rotation around $p$ bringing $\ell^{\prime}$ to coincide with $\ell$, minus $\frac{\pi}{2}$. Because of this definition, the slope of any half-line is between $-\frac{\pi}{2}$ (included) and $\frac{3 \pi}{2}$ (excluded); in the following there will be very few exceptions to this assumption, which will be however evident from the text. Every angle expressed as $\arctan (\cdot)$ is between $-\frac{\pi}{2}$ and $\frac{\pi}{2}$. We define the slope of an edge $u v$ in a graph drawing as the slope of the half-line from $u$ through $v$. Then the slope of an edge $u v$ is equal to the slope of the edge $v u$ plus or minus $\pi$. For a directed line $\ell$, we let its slope be equal to the slope of any half-line starting at a point of $\ell$ and directed as $\ell$. We denote by $\Delta p q r$ a triangle with vertices $p, q, r$, and we denote by $\measuredangle p q r$ the angle of $\Delta p q r$ incident to $q$; note that $\measuredangle p q r$ is between 0 and $\pi$.

Let $\Gamma$ be a drawing of a graph $G$ and let $u, v \in V(G)$. We denote by $d(\Gamma, u v)$ the Euclidean distance between the points representing $u$ and $v$ in $\Gamma$. We also denote by $d_{V}(\Gamma, u v)$ the vertical distance between $u$ and $v$ in $\Gamma$, that is, $d_{V}(\Gamma, u v)=|y(u)-y(v)|$, where the $y$ coordinates of $u$ and $v$ are taken from $\Gamma$; the horizontal distance $d_{H}(\Gamma, u v)$ between $u$ and $v$ in $\Gamma$ is defined analogously. With a slight abuse of notation, we will use $d(\Gamma, p q), d_{H}(\Gamma, p q)$, and $d_{V}(\Gamma, p q)$ even if $p$ and $q$ are points in the plane (and not vertices of $G$ ). A straight-line drawing of a graph is such that each edge is represented by a straight-line segment.

The following lemma argues that the planarity and the greediness of a drawing are not lost as a consequence of any sufficiently small perturbation of the vertex positions.

- Lemma 4. Let $\Gamma$ be a planar straight-line drawing of a graph $G$. There is a value $\varepsilon_{\Gamma}^{*}>0$ such that the following holds. Let $\Gamma^{\prime}$ be any straight-line drawing in which, for every vertex $z \in V(G)$, the Euclidean distance between the positions of $z$ in $\Gamma$ and $\Gamma^{\prime}$ is at most $\varepsilon_{\Gamma}^{*}$; then $\Gamma^{\prime}$ is planar and any path which is distance-decreasing in $\Gamma$ is also distance-decreasing in $\Gamma^{\prime}$. 


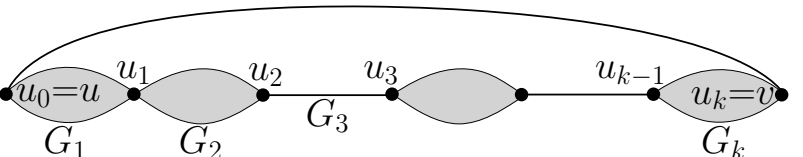

(a)

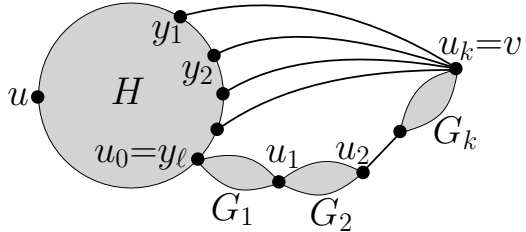

(b)

Figure 1 (a) Structure of $(G, u, v)$ in Case A. (b) Structure of $(G, u, v)$ in Case B.

\section{Proof of Theorem 3}

In this section we prove Theorem 3. Throughout the section, we will work with plane graphs. Further, we will deal with a class of graphs, known as strong circuit graphs [7], that is wider than 3-connected planar graphs. Strong circuit graphs constitute a subclass of the well-known circuit graphs, whose definition is due to Barnette and dates back to 1966 [5]. Here we rephrase the definition of strong circuit graphs as follows.

- Definition 5. A strong circuit graph is a triple $(G, u, v)$ such that either: (i) $G$ is an edge $u v$ or (ii) $|V(G)| \geq 3$ and the following properties are satisfied.

(a) $G$ is a 2-connected plane graph;

(b) $u$ and $v$ are two distinct external vertices of $G$;

(c) if edge $u v$ exists, then it coincides with the path $\tau_{u v}(G)$; and

(d) for every 2-cut $\{a, b\}$ of $G$ we have that $a$ and $b$ are external vertices of $G$ and at least one of them is an internal vertex of the path $\beta_{u v}(G)$; further, every non-trivial $\{a, b\}$-component of $G$ contains an external vertex of $G$ different from $a$ and $b$.

Several problems are easier to solve on (strong) circuit graphs than on 3-connected planar graphs. Indeed, (strong) circuit graphs can be easily decomposed into smaller (strong) circuit graphs and hence are suitable for inductive proofs. We now present a structural decomposition for strong circuit graphs whose main ideas can be found in a paper by Chen and $\mathrm{Yu}$ [7] (see [9] for an application of this decomposition to cubic strong circuit graphs).

Consider a strong circuit graph $(G, u, v)$ such that $G$ is not a single edge. The decomposition distinguishes the case in which the path $\tau_{u v}(G)$ coincides with the edge $u v$ (Case A) from the case in which it does not (Case B).

- Lemma 6. Suppose that we are in Case A (refer to Fig. 1(a)). Then the graph $G^{\prime}=G-u v$ consists of a sequence of graphs $G_{1}, \ldots, G_{k}$, with $k \geq 1$, such that:

- 6a: for $i=1, \ldots, k-1$, the graphs $G_{i}$ and $G_{i+1}$ share a single vertex $u_{i}$; further, $G_{i}$ is in the outer face of $G_{i+1}$ and vice versa in the plane embedding of $G$;

- 6b: for $1 \leq i, j \leq k$ with $j \geq i+2$, the graphs $G_{i}$ and $G_{j}$ do not share any vertex; and

- $6 c$ : for $i=1, \ldots, k$ with $u_{0}=u$ and $u_{k}=v,\left(G_{i}, u_{i-1}, u_{i}\right)$ is a strong circuit graph.

Given a strong circuit graph $(G, u, v)$ that is not a single edge, the vertex $u$ belongs to one 2-connected component of the graph $G-\{v\}$. Indeed, if it belonged to more than one 2-connected component of $G-\{v\}$, then $\{u\}$ would be a 1-cut of $G-\{v\}$, hence $\{u, v\}$ would be a 2-cut of $G$, which contradicts Property (d) for $(G, u, v)$. We now present the following.

- Lemma 7. Suppose that we are in Case B (refer to Fig. 1(b)). Let $H$ be the 2-connected component of the graph $G-\{v\}$ that contains $u$; then we have $|V(H)| \geq 3$. Let $H^{\prime}:=H \cup\{v\}$. Then $G$ contains $\ell$ distinct $H^{\prime}$-bridges $B_{1}, \ldots, B_{\ell}$, for some $\ell \geq 2$, such that: 
- 7a: each $H^{\prime}$-bridge $B_{i}$ has two attachments, namely $v$ and a vertex $y_{i} \in V(H)$;

- 7b: the $H^{\prime}$-bridges $B_{1}, \ldots, B_{\ell-1}$ are trivial, while $B_{\ell}$ might be trivial or not;

- 7 c: any two among $y_{1}, \ldots, y_{\ell}$ are distinct except, possibly, for $y_{\ell-1}$ and $y_{\ell}$; also if $\ell=2$, then $y_{1}$ and $y_{2}$ are distinct;

- 7d: $y_{1}$ is an internal vertex of $\tau_{u v}(G)$; further, $B_{1}$ is an edge that coincides with $\tau_{y_{1} v}(G)$;

- 7e: $y_{\ell}$ is an internal vertex of $\beta_{u v}(G)$ and $\beta_{u y_{1}}(H)$; further, $B_{\ell}$ contains the path $\beta_{y_{\ell} v}(G)$;

- 7f: $B_{1}, \ldots, B_{\ell-1}$ appear in this counter-clockwise order around $v$ and lie in the outer face of $B_{\ell}$ in the plane embedding of $G$;

- 7g: the triple $\left(H, u, y_{1}\right)$ is a strong circuit graph; and

- Th: $B_{\ell}$ consists of a sequence of graphs $G_{1}, \ldots, G_{k}$, with $k \geq 1$, such that:

- for $i=1, \ldots, k-1$, the graphs $G_{i}$ and $G_{i+1}$ share a single vertex $u_{i}$; further, $G_{i}$ is in the outer face of $G_{i+1}$ and vice versa in the plane embedding of $G$;

- for $1 \leq i, j \leq k$ with $j \geq i+2$, the graphs $G_{i}$ and $G_{j}$ do not share any vertex; and

- for $i=1, \ldots, k$ with $u_{0}=y_{\ell}$ and $u_{k}=v$, the triple $\left(G_{i}, u_{i-1}, u_{i}\right)$ is a strong circuit graph.

We prove that any strong circuit graph $(G, u, v)$ has a planar greedy drawing by exploiting Lemmata 6 and 7 in a natural way. Indeed, if we are in Case A (in Case B) then Lemma 6 (resp. Lemma 7 ) is applied in order to construct strong circuit graphs $\left(G_{i}, u_{i-1}, u_{i}\right)$ with $i=1, \ldots, k$ (resp. strong circuit graphs $\left(H, u, y_{1}\right)$ and $\left(G_{i}, u_{i-1}, u_{i}\right)$ with $\left.i=1, \ldots, k\right)$ for which planar greedy drawings are inductively constructed and combined together in order to get a planar greedy drawing of $(G, u, v)$. The base case of the induction is the one in which $G$ is an edge; then a planar greedy drawing of $G$ is directly constructed. In order to be able to combine the planar greedy drawings for the strong circuit graphs $\left(G_{i}, u_{i-1}, u_{i}\right)$ (and $\left(H, u, y_{1}\right)$ if we are in Case B) to construct a planar greedy drawing of $(G, u, v)$, we need the inductively constructed drawings to satisfy some restrictive geometric requirements. These are expressed in the following theorem, which is the core of the proof of Theorem 3.

- Theorem 8. Let $(G, u, v)$ be a strong circuit graph with at least three vertices and let $0<\alpha<\frac{\pi}{4}$ be an arbitrary parameter. Let $\beta_{u v}(G)=\left(u=b_{1}, b_{2}, \ldots, b_{m}=v\right)$. There exists a straight-line drawing $\Gamma$ of $G$ in the Cartesian plane such that the following holds. For any value $\delta \geq 0$, denote by $\Gamma_{\delta}$ the straight-line drawing obtained from $\Gamma$ by moving the position of vertex $u$ by $\delta$ units to the left. Then $\Gamma_{\delta}$ satisfies the following properties (refer to Fig. 2).

1. $\Gamma_{\delta}$ is planar;

2. $\tau_{u v}(G)$ lies entirely on a horizontal line $\ell_{u}$ with $u$ to the left of $v$;

3. the edge $b_{1} b_{2}$ has slope in the interval $(-\alpha, 0)$ and the edge $b_{i} b_{i+1}$ has slope in the interval $(0, \alpha)$, for each $i=2,3, \ldots, m-1$;

4. for every vertex $x \in V(G)$ there is a path $P_{x}=\left(x=v_{1}, v_{2}, \ldots, v_{p}=v\right)$ from $x$ to $v$ in $G$ such that the edge $v_{i} v_{i+1}$ has slope in the interval $(-\alpha, \alpha)$ in $\Gamma_{\delta}$, for each $i=1,2, \ldots, p-1$; further, if $x \neq u$, then $u \notin V\left(P_{x}\right)$;

5. for every vertex $x \in V(G)$ there is a path $Q_{x}=\left(x=w_{1}, w_{2}, \ldots, w_{q}=u\right)$ from $x$ to $u$ in $G$ such that the edge $w_{i} w_{i+1}$ has slope in the interval $(\pi-\alpha, \pi+\alpha)$ in $\Gamma_{\delta}$, for each $i=1,2, \ldots, q-1 ;$ and

6. for every ordered pair of vertices $(x, y)$ in $V(G)$ there is a path $P_{x y}$ from $x$ to $y$ in $G$ such that $P_{x y}$ is distance-decreasing in $\Gamma_{\delta}$; further, if $x \neq u$ and $y \neq u$, then $u \notin V\left(P_{x y}\right)$.

We comment on the statement of Theorem 8. First, let us set $\delta=0$ and argue about $\Gamma_{0}=\Gamma$. Properties 1 and 6 are those that one would expect, as they state that $\Gamma$ is planar and greedy, respectively. Properties 2 and 3 state that all the edges incident to the outer face of $\Gamma$ are "almost" horizontal; indeed, the edges of $\tau_{u v}(G)$ are horizontal (this does not 


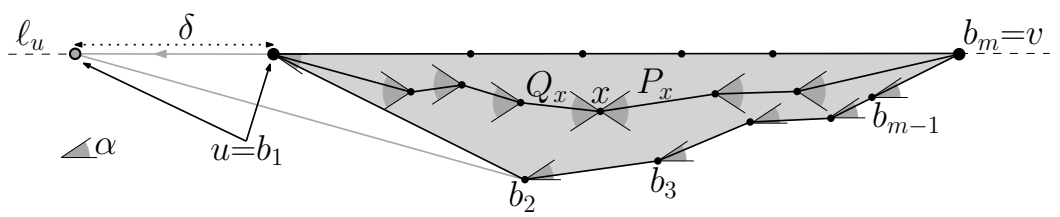

Figure 2 Illustration for the statement of Theorem 8.

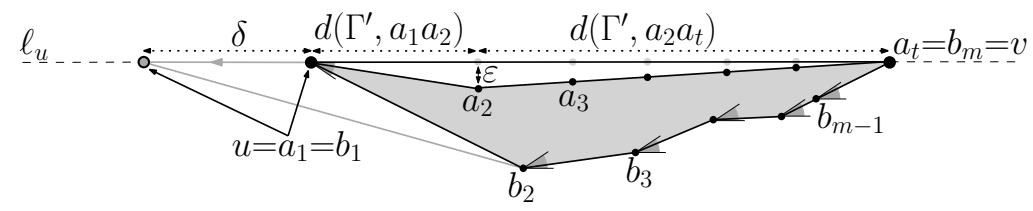

Figure 3 The straight-line drawing $\Gamma$ of $G$ in Case A if $k=1$.

compromise the planarity of $\Gamma$ since $G$ does not contain edges between non-consecutive vertices of $\tau_{u v}(G)$, by Property (d) of $\left.(G, u, v)\right)$, the edge $b_{1} b_{2}$ has a slightly negative slope, and all the other edges of $\beta_{u v}(G)$ have slightly positive slopes. Then the planarity of $\Gamma$ implies that $\Gamma$ is contained in a wedge delimited by two half-lines with slopes 0 and $-\alpha$ starting at $u$. Properties 4 and 5 argue about the existence of certain paths from any vertex to $u$ and $v$; these two vertices play an important role in the structural decomposition we employ, since distinct subgraphs are joined on those vertices, and the paths incident to them are inductively combined together in order to construct distance-decreasing paths. Finally, all these properties still hold if $u$ is moved by an arbitrary non-negative amount $\delta$ to the left. This is an important feature we exploit in one of our inductive cases.

We now present an inductive proof of Theorem 8. In the Base Case the graph $G$ is a single edge. We remark that, although Theorem 8 assumes that the given graph has at least three vertices, for its proof we need to inductively draw certain subgraphs of it; these subgraphs might indeed be single edges. Whenever we need to draw a strong circuit graph $(G, u, v)$ such that $G$ is a single edge $u v$, we draw it as a horizontal straight-line segment with positive length, with $u$ to the left of $v$. We remark that, since Theorem 8 assumes that $|V(G)| \geq 3$, we do not need the constructed drawing to satisfy Properties $1-6$.

We now discuss the inductive cases. In Case A the path $\tau_{u v}(G)$ coincides with the edge $u v$, while in Case B it does not. We discuss Case A first. Let $G^{\prime}=G-u v$, where $G^{\prime}$ consists of a sequence of graphs $G_{1}, \ldots, G_{k}$, with $k \geq 1$, satisfying the properties described in Lemma 6. Our construction is different if $k=1$ and $k \geq 2$.

Suppose first that $\mathbf{k}=\mathbf{1}$; by Lemma 6 the triple $\left(G^{\prime}=G_{1}, u, v\right)$ is a strong circuit graph (and $G_{1}$ is not a single edge, as otherwise we would be in the Base Case). Inductively construct a straight-line drawing $\Gamma^{\prime}$ of $G^{\prime}$ with $\frac{\alpha}{2}$ as a parameter. By Property 2 the path $\tau_{u v}\left(G^{\prime}\right)=\left(u=a_{1}, \ldots, a_{t}=v\right)$ lies on a horizontal line $\ell_{u}$ in $\Gamma^{\prime}$ with $u$ to the left of $v$. Let $Y>0$ be the minimum distance in $\Gamma^{\prime}$ of any vertex strictly below $\ell_{u}$ from $\ell_{u}$. Let

$$
\varepsilon=\frac{1}{2} \min \left\{\varepsilon_{\Gamma^{\prime}}^{*}, Y, \tan (\alpha) \cdot d\left(\Gamma^{\prime}, a_{1} a_{2}\right), \tan (\alpha) \cdot d\left(\Gamma^{\prime}, a_{2} a_{t}\right)\right\} .
$$

We construct a straight-line drawing $\Gamma$ of $G$ from $\Gamma^{\prime}$ as follows; refer to Fig. 3. Decrease the $y$-coordinate of the vertex $a_{2}$ by $\varepsilon$; for $i=3, \ldots, t-1$, decrease the $y$-coordinate of the vertex $a_{i}$ so that it ends up on the straight-line segment $\overline{a_{2} a_{t}}$. Draw the edge $u v$ as a straight-line segment. We have the following. 


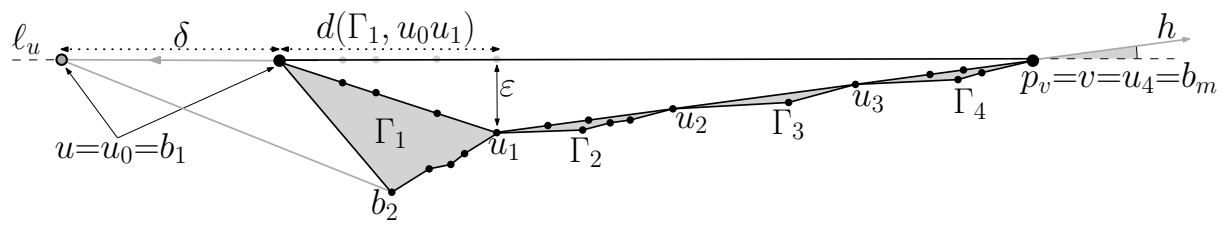

Figure 4 The straight-line drawing $\Gamma$ of $G$ in Case $\mathrm{A}$ if $k \geq 2$. In this example $k=4$. The gray angle in the drawing is $\frac{\alpha}{2}$.

Lemma 9. For any $\delta \geq 0$, the drawing $\Gamma_{\delta}$ constructed in Case $A$ if $k=1$ satisfies Properties 1-6 of Theorem 8.

Proof Sketch. The planarity of $\Gamma$ is established due to the inequality $\varepsilon<\varepsilon_{\Gamma^{\prime}}^{*}$ and to Lemma 4 . Since $\Gamma$ and $\Gamma_{\delta}$ coincide, except for the position of $u$, every crossing in $\Gamma_{\delta}$ has to involve edges incident to $u$. The proof that in fact there are no such crossings relies on the fact that $\Gamma_{\delta}^{\prime}$ is planar, by induction, and on the inequalities $\varepsilon<\varepsilon_{\Gamma^{\prime}}^{*}$ and $\varepsilon<Y$.

The paths $P_{x}$ and $Q_{x}$ requested for Properties 4 and 5 are obtained by suitably modifying paths satisfying the same properties for $\left(G^{\prime}, u, v\right)$. The paths $P_{x}$ and $Q_{x}$ might contain edges in $\tau_{u v}\left(G^{\prime}\right)$; however, the slopes of the edges are in the required interval, which is $(-\alpha, \alpha)$ or $(\pi-\alpha, \pi+\alpha)$ depending on whether these edges are traversed towards $v$ or $u$, respectively. This is due to the inequalities $\varepsilon<\tan (\alpha) \cdot d\left(\Gamma^{\prime}, a_{1} a_{2}\right)$ and $\varepsilon<\tan (\alpha) \cdot d\left(\Gamma^{\prime}, a_{2} a_{t}\right)$.

We now discuss the case in which $\mathbf{k} \geq \mathbf{2}$. Refer to Fig. 4. By Lemma 6 , for $i=1, \ldots, k$, the triple $\left(G_{i}, u_{i-1}, u_{i}\right)$ is a strong circuit graph, where $u_{0}=u, u_{k}=v$, and $u_{i}$ is the only vertex shared by $G_{i}$ and $G_{i+1}$, for $i=1, \ldots, k-1$.

If $G_{1}$ is a single edge, then inductively construct a straight-line drawing $\Gamma_{1}$ of $G_{1}$ and define $\varepsilon=\frac{1}{2} \min \left\{\varepsilon_{\Gamma_{1}}^{*}, \tan (\alpha) \cdot d\left(\Gamma_{1}, u_{0} u_{1}\right)\right\}$. If $G_{1}$ is not a single edge, then inductively construct a straight-line drawing $\Gamma_{1}$ of $G_{1}$ with $\frac{\alpha}{2}$ as a parameter. By Property 2 of $\Gamma_{1}$, the path $\tau_{u_{0} u_{1}}\left(G_{1}\right)$ lies on a horizontal line $\ell_{u}$. Let $Y>0$ be the minimum distance in $\Gamma_{1}$ of any vertex strictly below $\ell_{u}$ from $\ell_{u}$. Let $\varepsilon=\frac{1}{2} \min \left\{\varepsilon_{\Gamma_{1}}^{*}, Y, \tan (\alpha) \cdot d\left(\Gamma_{1}, u_{0} u_{1}\right)\right\}$. In both cases, decrease the $y$-coordinate of $u_{1}$ by $\varepsilon$. Further, decrease the $y$-coordinate of every internal vertex of the path $\tau_{u_{0} u_{1}}\left(G_{1}\right)$, if any, so that it ends up on the straight-line segment $\overline{u_{0} u_{1}}$.

Now consider a half-line $h$ with slope $s=\frac{\alpha}{2}$ starting at $u_{1}$. Denote by $p_{v}$ the point at which $h$ intersects the horizontal line $\ell_{u}$ through $u$. For $i=2, \ldots, k$, inductively construct a straight-line drawing $\Gamma_{i}$ of $G_{i}$ with $\frac{\alpha}{3}$ as a parameter (if $G_{i}$ is a single edge, then the parameter does not matter). Uniformly scale the drawings $\Gamma_{2}, \ldots, \Gamma_{k}$ so that the Euclidean distance between $u_{i-1}$ and $u_{i}$ in $\Gamma_{i}$ is equal to $\frac{d\left(\Gamma_{1}, u_{1} p_{v}\right)}{k-1}$. For $i=2, \ldots, k$, rotate the scaled drawing $\Gamma_{i}$ around $u_{i-1}$ counter-clockwise by $s$ radians. Translate the scaled and rotated drawings $\Gamma_{2}, \ldots, \Gamma_{k}$ so that the representations of $u_{i}$ in $\Gamma_{i}$ and $\Gamma_{i+1}$ coincide, for $i=1, \ldots, k-1$. Finally, draw the edge $u v$ as a straight-line segment. This completes the construction of a drawing $\Gamma$ of $G$. We have the following.

- Lemma 10. For any $\delta \geq 0$, the drawing $\Gamma_{\delta}$ constructed in Case $A$ if $k \geq 2$ satisfies Properties 1-6 of Theorem 8.

Proof Sketch. The fulfillment of Property 3 for $\Gamma_{\delta}$ is the reason for the asymmetry of the construction, which shifts vertices in $\Gamma_{1}$, while it rotates $\Gamma_{2}, \ldots, \Gamma_{k}$. Indeed, for $i=1, \ldots, k$, the first edge of $\beta_{u_{i-1} u_{i}}\left(G_{i}\right)$ has negative slope in $\Gamma_{i}$, while all the other edges have positive slopes; we need to ensure that the same property holds for $\beta_{u v}(G)=\beta_{u_{0} u_{1}}\left(G_{1}\right) \cup \cdots \cup$ $\beta_{u_{k-1} u_{k}}\left(G_{k}\right)$ in $\Gamma_{\delta}$. For $i=2, \ldots, k$, the counter-clockwise rotation of $\Gamma_{i}$ by $s=\frac{\alpha}{2}$ radians 


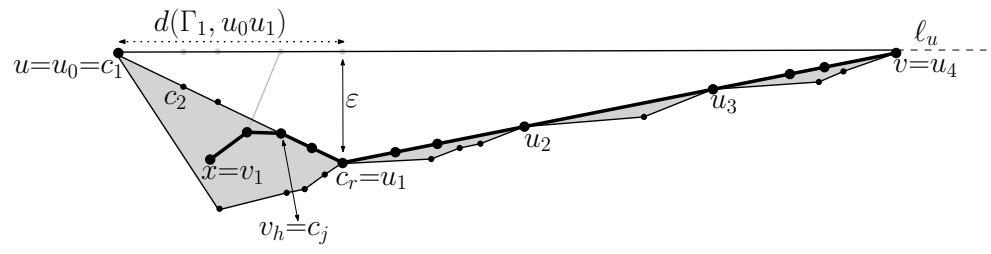

Figure 5 Illustration for the proof that the slope in $\Gamma_{\delta}$ of every edge in the path $P_{x}$ is in $(-\alpha, \alpha)$, in the case in which $x$ belongs to $G_{1}$. The path $P_{x}$ is thick.

makes up for the negative slope (at most $\frac{\alpha}{3}$ in absolute value) of the first edge of $\beta_{u_{i-1} u_{i}}\left(G_{i}\right)$ in $\Gamma_{i}$. On the other hand, the edges of $\beta_{u_{0} u_{1}}\left(G_{1}\right)$ do not move when transforming $\Gamma_{1}$ in $\Gamma$, except for the edge incident to $u_{1}$, which however does not change its slope significantly, due to the inequality $\varepsilon<Y$; hence the slope of the first edge of $\beta_{u_{0} u_{1}}\left(G_{1}\right)$ remains negative (or becomes negative if $G_{1}$ is a single edge) and the other ones remain positive.

We present a proof that $\Gamma_{\delta}$ satisfies Property 4 . Let $x \in V(G)$. If $x=u$, let $P_{x}=(u, v)$; then the only edge of $P_{x}$ has slope $0 \in(-\alpha, \alpha)$ in $\Gamma_{\delta}$. If $x=u_{i}$, for some $i \in\{1, \ldots, k-1\}$, then let $P_{x}=\bigcup_{j=i+1}^{k} \tau_{u_{j-1} u_{j}}\left(G_{j}\right)$ and observe that all the edges of $P_{x}$ have slope $s=\frac{\alpha}{2} \in(-\alpha, \alpha)$; further $P_{x}$ does not pass through $u$. If $x \neq u_{i}$, for every $i \in\{0, \ldots, k\}$, then $x$ belongs to a unique graph $G_{i}$, for some $i \in\{1, \ldots, k\}$. Assume that $i=1$; the case $i \geq 2$ is easier to handle. Refer to Fig. 5. Let $\tau_{u_{0} u_{1}}\left(G_{1}\right)=\left(u_{0}=c_{1}, c_{2}, \ldots, c_{r}=u_{1}\right)$. Since $\Gamma_{1}$ satisfies Property 4 , there exists a path $P_{x}^{1}=\left(x=v_{1}, v_{2}, \ldots, v_{p}=u_{1}\right)$ from $x$ to $u_{1}$ in $G_{1}$, not passing through $u_{0}$, whose edges have slopes in $\left(-\frac{\alpha}{2}, \frac{\alpha}{2}\right)$ in $\Gamma_{1}$; let $h$ be the smallest index such that $v_{h}=c_{j}$, for some $j \in\{1, \ldots, r\}$. Such an index $h$ exists (possibly $h=p$ and $j=r$ ). Then let $P_{x}$ consist of the paths $\left(x=v_{1}, v_{2}, \ldots, v_{h}\right),\left(v_{h}=c_{j}, c_{j+1}, \ldots, c_{r}\right)$, and $\bigcup_{j=2}^{k} \tau_{u_{j-1} u_{j}}\left(G_{j}\right)$. Since $u \notin V\left(P_{x}^{1}\right)$, we have that $u \notin V\left(P_{x}\right)$, hence it suffices to argue about the slopes of the edges of $P_{x}$ in $\Gamma$ rather than in $\Gamma_{\delta}$.

For $l=1, \ldots, h-2$, the slope of $v_{l} v_{l+1}$ is in $(-\alpha, \alpha)$ in $\Gamma$ since it is in $(-\alpha, \alpha)$ in $\Gamma_{1}$ and since neither $v_{l}$ nor $v_{l+1}$ moves when transforming $\Gamma_{1}$ into $\Gamma$. Further, for $l=j, \ldots, r-1$, the slope of the edge $c_{l} c_{l+1}$ in $\Gamma$ is $-\arctan \left(\frac{\varepsilon}{d\left(\Gamma_{1}, u_{0} u_{1}\right)}\right)$, which is in the interval $(-\alpha, 0) \subset(-\alpha, \alpha)$, given that $\varepsilon, d\left(\Gamma_{1}, u_{0} u_{1}\right)>0$ and that $\varepsilon<\tan (\alpha) \cdot d\left(\Gamma_{1}, u_{0} u_{1}\right)$. Moreover, the edges of $\bigcup_{j=2}^{k} \tau_{u_{j-1} u_{j}}\left(G_{j}\right)$ have slope $s=\frac{\alpha}{2} \in(-\alpha, \alpha)$. Finally, let $\sigma_{1}$ and $\sigma$ be the slopes of the edge $v_{h-1} v_{h}$ in $\Gamma_{1}$ and $\Gamma$, respectively. Since $v_{h-1} v_{h} \in E\left(P_{x}^{1}\right)$, we have $\sigma_{1} \in\left(-\frac{\alpha}{2}, \frac{\alpha}{2}\right) ;$ since $\alpha \leq \frac{\pi}{4}$, we have $x\left(v_{h-1}\right)<x\left(v_{h}\right)$ in $\Gamma_{1}$ and $\Gamma$ (note that the $x$-coordinates of the vertices do not change when transforming $\Gamma_{1}$ into $\Gamma$ ). Further, by Properties $1-4$ of $\Gamma_{1}$, we have that $v_{h-1}$ lies below $\ell_{u}$, which contains $v_{h}$; hence, $y\left(v_{h-1}\right)<y\left(v_{h}\right)$ in $\Gamma_{1}$. Since the vertex $v_{h}$ moves down (while $v_{h-1}$ stays put) when transforming $\Gamma_{1}$ into $\Gamma$, and since $\varepsilon \leq \frac{Y}{2}<d_{V}\left(\Gamma_{1}, v_{h-1} v_{h}\right)$, it follows that $0<\sigma<\sigma_{1}$; hence $\sigma \in\left(0, \frac{\alpha}{2}\right) \subset(-\alpha, \alpha)$.

Turning our attention to Property 6 , consider any two vertices $x, y \in V(G)$, and assume that $x \in V\left(G_{i}\right)$ and $y \in V\left(G_{j}\right)$. We prove the existence of a path $P_{x y}$ from $x$ to $y$ in $G$ that is distance-decreasing in $\Gamma_{\delta}$ in the case in which $2 \leq i<j \leq k$; the other cases can be treated similarly. Let $P_{x y}$ consist of a path $P_{x}^{i}$ in $G_{i}$ from $x$ to $u_{i}$ whose edges have slopes in $\left(-\frac{\alpha}{3}, \frac{\alpha}{3}\right)$ in $\Gamma_{i}$, of the path $\bigcup_{l=i+1}^{j-1} \tau_{u_{l-1} u_{l}}\left(G_{l}\right)$, and of a path $P_{u_{j-1} y}^{j}$ in $G_{j}$ that is distancedecreasing in $\Gamma_{j}$. By induction, $P_{x}^{i}$ and $P_{u_{j-1} y}^{j}$ exist since $\Gamma_{i}$ and $\Gamma_{j}$ satisfy Properties 4 and 6, respectively; further, note that $u \notin V\left(P_{x y}\right)$. Let $P_{x y}=\left(z_{1}, z_{2}, \ldots, z_{s}\right)$; we prove that $d\left(\Gamma_{\delta}, z_{h} z_{s}\right)>d\left(\Gamma_{\delta}, z_{h+1} z_{s}\right)$, for $h=1,2, \ldots, s-2$, hence $P_{x y}$ is distance-decreasing in $\Gamma_{\delta}$. We distinguish three cases.

If $z_{h} z_{h+1}$ is in $G_{j}$, then $\left(z_{h}, z_{h+1}, \ldots, z_{s}\right)$ is a sub-path of $P_{u_{j-1} y}^{j}$, hence it is distancedecreasing in $\Gamma_{\delta}$ since it is distance-decreasing in $\Gamma_{j}$ and since the drawing of $G_{j}$ in $\Gamma_{\delta}$ is 


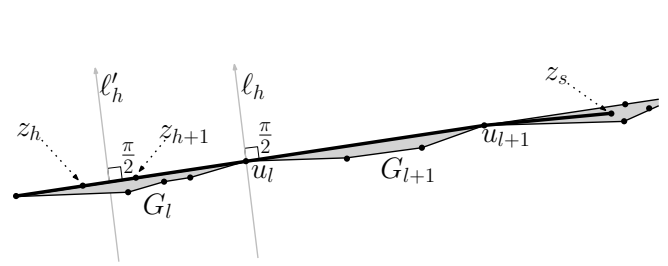

(a)

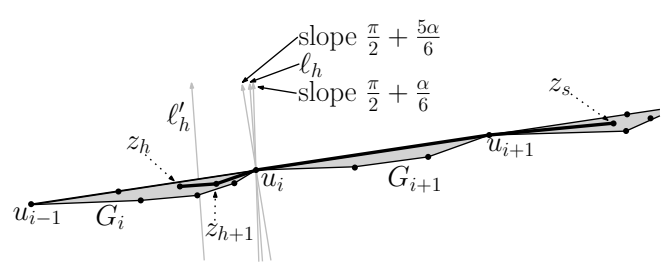

(b)

Figure 6 (a) Illustration for the proof that $d\left(\Gamma_{\delta}, z_{h} z_{s}\right)>d\left(\Gamma_{\delta}, z_{h+1} z_{s}\right)$ if $z_{h} z_{h+1}$ is in $\tau_{u_{l-1} u_{l}}\left(G_{l}\right)$. (b) Illustration for the proof that $d\left(\Gamma_{\delta}, z_{h} z_{s}\right)>d\left(\Gamma_{\delta}, z_{h+1} z_{s}\right)$ if $z_{h} z_{h+1}$ is in $P_{x}^{i}$.

congruent to $\Gamma_{j}$, up to affine transformations (a uniform scaling, a rotation, and a translation), which preserve the property of a path to be distance-decreasing.

If $z_{h} z_{h+1}$ is in $\tau_{u_{l-1} u_{l}}\left(G_{l}\right)$, for some $l \in\{i+1, i+2, \ldots, j-1\}$, as in Fig. $6(\mathrm{a})$, then it has slope $s=\frac{\alpha}{2}$. The directed line $\ell_{h}$ with slope $\frac{\pi+\alpha}{2}$ through $u_{l}$, oriented towards increasing $y$-coordinates has the drawings of $G_{l+1}, \ldots, G_{k}$ (and in particular the vertex $z_{s}$ ) to its right; this is because by Property 3 of $\Gamma_{\delta}$ every edge in $\beta_{u_{l} v}(G)$ has slope in the interval $(0, \alpha)$, where $\frac{-\pi+\alpha}{2}<0<\alpha<\frac{\pi+\alpha}{2}$, and because the path $\bigcup_{m=l+1}^{k} \tau_{u_{m-1} u_{m}}\left(G_{m}\right)$ has slope $s=\frac{\alpha}{2}$, where $\frac{-\pi+\alpha}{2}<\frac{\alpha}{2}<\frac{\pi+\alpha}{2}$. Then the directed line $\ell_{h}^{\prime}$ parallel to $\ell_{h}$, passing through the midpoint of the edge $z_{h} z_{h+1}$, and oriented towards increasing $y$-coordinates has $\ell_{h}$ to its right, hence it has $z_{s}$ to its right. Since the half-plane to the right of $\ell_{h}^{\prime}$ represents the locus of the points of the plane that are closer to $z_{h+1}$ than to $z_{h}$, it follows that $d\left(\Gamma_{\delta}, z_{h} z_{s}\right)>d\left(\Gamma_{\delta}, z_{h+1} z_{s}\right)$.

If $z_{h} z_{h+1}$ is in $P_{x}^{i}$, as in Fig. 6(b), then by Property 4 it has slope in $\left(-\frac{\alpha}{3}, \frac{\alpha}{3}\right)$ in $\Gamma_{i}$. Since $\Gamma_{i}$ is counter-clockwise rotated by $s$ radians in $\Gamma_{\delta}$, it follows that $z_{h} z_{h+1}$ has slope in $\left(s-\frac{\alpha}{3}, s+\frac{\alpha}{3}\right)=\left(\frac{\alpha}{6}, \frac{5 \alpha}{6}\right)$ in $\Gamma_{\delta}$. Consider the directed line $\ell_{h}$ that passes through $u_{i}$, that is directed towards increasing $y$-coordinates and that is orthogonal to the line through $z_{h}$ and $z_{h+1}$. Denote by $s_{h}$ the slope of $\ell_{h}$. Then $s_{h} \in\left(\frac{\pi}{2}+\frac{\alpha}{6}, \frac{\pi}{2}+\frac{5 \alpha}{6}\right)$. We have that $\ell_{h}$ has the drawings of $G_{i+1}, \ldots, G_{k}$ to its right; this is because by Property 3 of $\Gamma_{\delta}$ every edge in $\beta_{u_{i} v}(G)$ has slope in $(0, \alpha)$ with $s_{h}-\pi<-\frac{\pi}{2}+\frac{5 \alpha}{6}<0<\alpha<\frac{\pi}{2}+\frac{\alpha}{6}<s_{h}$ and because the path $\bigcup_{m=i+1}^{k} \tau_{u_{m-1} u_{m}}\left(G_{m}\right)$ has slope $s=\frac{\alpha}{2}$, where $s_{h}-\pi<-\frac{\pi}{2}+\frac{5 \alpha}{6}<\frac{\alpha}{2}<\frac{\pi}{2}+\frac{\alpha}{6}<s_{h}$. Further, $\ell_{h}$ has the drawings of $G_{2}, \ldots, G_{i}$ to its left; this is because by Property 3 of $\Gamma_{\delta}$ every edge in $\tau_{u_{i} u_{1}}(G)$ has slope in $(\pi, \pi+\alpha)$ with $s_{h}<\frac{\pi}{2}+\frac{5 \alpha}{6}<\pi<\pi+\alpha<\frac{3 \pi}{2}+\frac{\alpha}{6}<\pi+s_{h}$ and because the path $\bigcup_{m=2}^{i} \beta_{u_{m} u_{m-1}}\left(G_{m}\right)$ has slope $s=\pi+\frac{\alpha}{2}$, where $s_{h}<\frac{\pi}{2}+\frac{5 \alpha}{6}<\pi+\frac{\alpha}{2}<$ $\frac{3 \pi}{2}+\frac{\alpha}{6}<\pi+s_{h}$. Now consider the directed line $\ell_{h}^{\prime}$ parallel to $\ell_{h}$, passing through the midpoint of the edge $z_{h} z_{h+1}$, and oriented towards increasing $y$-coordinates. This line has $\ell_{h}$ to its right, given that the drawing of $G_{i}$ (and in particular the midpoint of $z_{h} z_{h+1}$ ) is to the left of $\ell_{h}$ in $\Gamma_{\delta}$. Thus, $\ell_{h}^{\prime}$ has the drawings of $G_{l+1}, \ldots, G_{k}$ (and in particular the vertex $z_{s}$ ) to its right. Since the half-plane to the right of $\ell_{h}^{\prime}$ represents the locus of the points of the plane that are closer to $z_{h+1}$ than to $z_{h}$, it follows that $d\left(\Gamma_{\delta}, z_{h} z_{s}\right)>d\left(\Gamma_{\delta}, z_{h+1} z_{s}\right)$.

We now discuss Case B, in which $(G, u, v)$ is decomposed according to Lemma 7. Refer to Figs. 7 and 8. First, the triple $\left(H, u, y_{1}\right)$ is a strong circuit graph with $|V(H)| \geq 3$. Inductively construct a straight-line drawing $\Gamma_{H}$ of $H$ with $\frac{\alpha}{2}$ as a parameter.

Let $\beta_{u y_{1}}(H)=\left(u=b_{1}, \ldots, b_{m}=y_{1}\right)$. Let $\phi_{i}$ be the slope of the edge $b_{i} b_{i+1}$ in $\Gamma_{H}$ and let $\phi=\min _{i=2, \ldots, m-1}\left\{\phi_{i}\right\}$. By Property (c) of $\left(H, u, y_{1}\right)$ if the edge $u y_{1}$ belongs to $H$ then it coincides with the path $\tau_{u y_{1}}(H)$. Hence, $m \geq 3$ and $\phi$ is well-defined. Further, $\phi$ is in the interval $\left(0, \frac{\alpha}{2}\right)$ by Property 3 of $\Gamma_{H}$. 


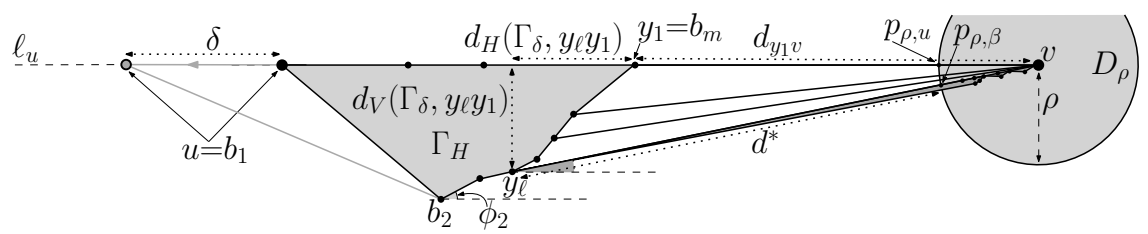

Figure 7 The straight-line drawing $\Gamma$ of $G$ in Case B. For the sake of readability, $\phi$ and $\rho$ are larger than they should be. The dark gray angle is $\beta$. Fig. 8 shows an enlarged view of $D_{\rho}$.

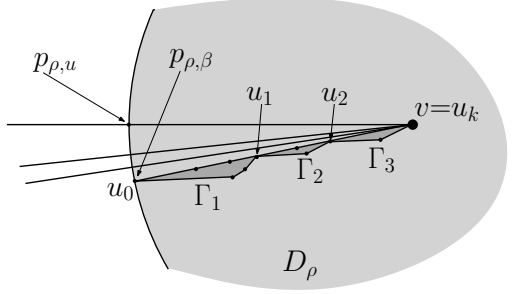

(a)

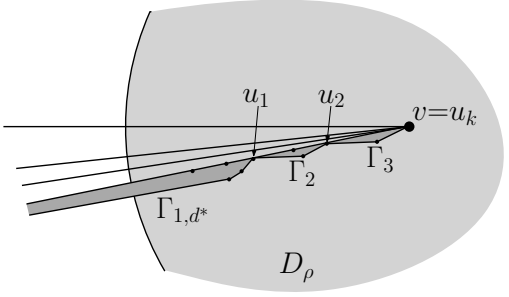

(b)

Figure $8 \mathrm{~A}$ closer look at $D_{\rho}$. Figure (a) represents the drawings $\Gamma_{1}, \ldots, \Gamma_{k}$ once they have been uniformly scaled, rotated, and translated, while (b) also has the vertex $u_{0}$ moved by $d^{*}$ units (this movement actually happens before the rotation and translation of $\Gamma_{1}$ ).

Let $\beta=\frac{1}{2} \min \left\{\phi, \arctan \left(\frac{d_{V}\left(\Gamma_{H}, y_{\ell} y_{1}\right)}{3 d_{V}\left(\Gamma_{H}, y_{\ell} y_{1}\right)+3 d_{H}\left(\Gamma_{H}, y_{\ell} y_{1}\right)}\right)\right\}$. Note that $\beta>0$, given that $\phi, d_{V}\left(\Gamma_{H}, y_{\ell} y_{1}\right)>0$ and $d_{H}\left(\Gamma_{H}, y_{\ell} y_{1}\right) \geq 0$. In particular, $d_{V}\left(\Gamma_{H}, y_{\ell} y_{1}\right)>0$ because $y_{1}$ is a vertex of $\tau_{u y_{1}}(H)$ and $y_{\ell}$ is an internal vertex of $\beta_{u y_{1}}(H)$ by Lemma 7 , and because of Properties 1-3 of $\Gamma_{H}$. Also note that $\beta<\frac{\alpha}{4}$, given that $\phi<\frac{\alpha}{2}$.

Consider a half-line $h_{\beta}$ with slope $\beta$ starting at $y_{\ell}$. Place the vertex $v$ at the intersection point between $h_{\beta}$ and the horizontal line $\ell_{u}$ through $u$. Draw all the trivial $(H \cup\{v\})$-bridges of $G$ as straight-line segments. This concludes the construction if every $(H \cup\{v\})$-bridge of $G$ is trivial. Otherwise, $B_{\ell}$ is the only non-trivial $(H \cup\{v\})$-bridge of $G$. Then $B_{\ell}$ consists of $k$ strong circuit graphs $\left(G_{i}, u_{i-1}, u_{i}\right)$, where $u_{0}=y_{\ell}$ and $u_{k}=v$. With a slight change of notation, in the remainder of the section we assume that, if the edge $y_{\ell} v$ exists, then it is an edge of $B_{\ell}$ (rather than an individual trivial $\left(H \cup\{v\}\right.$ )-bridge $B_{\ell-1}$ of $G$ ); in this case $\left(B_{\ell}, u_{0}, u_{k}\right)$ is a strong circuit graph.

We claim that $v$ lies to the right of $y_{1}$. The polygonal line representing $\beta_{y_{\ell} y_{1}}(H)$ in $\Gamma_{H}$ and the straight-line segment $\overline{y_{\ell} v}$ are both incident to $y_{\ell}$. By definition of $\phi$ and since $\Gamma_{H}$ satisfies Property $3, \beta_{y_{\ell} y_{1}}(H)$ is composed of straight-line segments with slopes in the range $\left[\phi, \frac{\alpha}{2}\right)$, while $\overline{y_{\ell} v}$ has slope $\beta$. The claim then follows from $0<\beta<\phi<\frac{\pi}{2}$. Let $d_{y_{1} v}$ be the distance between $y_{1}$ and $v$. Let $Y>0$ be the minimum distance in $\Gamma_{H}$ of any vertex strictly below $\ell_{u}$ from $\ell_{u}$. Let $\rho=\min \left\{\frac{d_{y_{1} v}}{3}, \frac{Y}{2}\right\}$ and let $D_{\rho}$ be the disk with radius $\rho$ centered at $v$. Let $p_{\rho, \beta}\left(p_{\rho, u}\right)$ be the intersection point of the boundary of $D_{\rho}$ with $h_{\beta}$ (resp. with $\ell_{u}$ ) that is closer to $y_{\ell}$ (resp. to $y_{1}$ ). Let $d^{*}$ be the Euclidean distance between $y_{\ell}$ and $p_{\rho, \beta}$.

Let $\alpha^{\prime}=\frac{\beta}{2}$. Since $\beta>0$, we have $\alpha^{\prime}>0$; further, $\alpha^{\prime}<\frac{\alpha}{8}$, given that $\beta<\frac{\alpha}{4}$. For $i=1, \ldots, k$, inductively construct a straight-line drawing $\Gamma_{i}$ of $G_{i}$ with $\alpha^{\prime}$ as a parameter (if $G_{i}$ is a single edge, then the parameter does not matter). Uniformly scale the drawings $\Gamma_{1}, \ldots, \Gamma_{k}$ so that the Euclidean distance between $u_{i-1}$ and $u_{i}$ is equal to $\frac{\rho}{k}$. Move the vertex $u_{0}$ in $\Gamma_{1}$ by $d^{*}$ units to the left, obtaining a drawing $\Gamma_{1, d^{*}}$. Rotate the drawings $\Gamma_{1, d^{*}}, \Gamma_{2}, \ldots, \Gamma_{k}$ counter-clockwise by $\beta$ radians. Translate $\Gamma_{1, d^{*}}, \Gamma_{2}, \ldots, \Gamma_{k}$ so that, for $i=1, \ldots, k-1$, the representations of $u_{i}$ in $\Gamma_{i}$ and $\Gamma_{i+1}$ (in $\Gamma_{1, d^{*}}$ and $\Gamma_{2}$ if $i=1$ ) coincide 


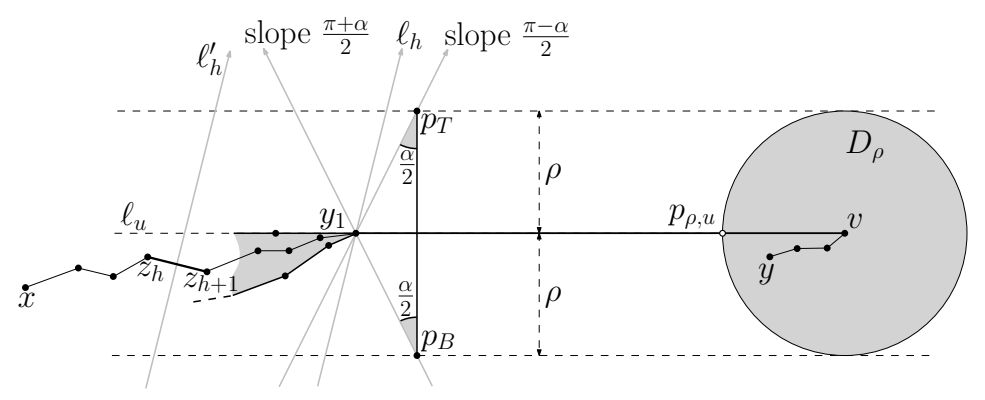

Figure 9 Illustration for the proof that $d\left(\Gamma_{\delta}, z_{h} y\right)>d\left(\Gamma_{\delta}, z_{h+1} y\right)$ if $z_{h} z_{h+1}$ is in $P_{x}^{H}$. For the sake of readability, $D_{\rho}$ is larger than it should be.

and so that the representation of $u_{0}$ in $\Gamma_{1, d^{*}}$ coincides with the one of $y_{\ell}$ in $\Gamma_{H}$. This completes the construction of a straight-line drawing $\Gamma$ of $G$. We have the following.

- Lemma 11. For any $\delta \geq 0$, the drawing $\Gamma_{\delta}$ constructed in Case B satisfies Properties 1-6 of Theorem 8 .

Proof Sketch. Let $\Gamma_{H, \delta}$ denote the drawing obtained from $\Gamma_{H}$ by moving the vertex $u$ by $\delta$ units to the left.

We first prove that every vertex $z \neq u_{0}$ that belongs to a graph $G_{i}$ lies inside the disk $D_{\rho}$ in $\Gamma_{\delta}$. A consequence of this statement is a sharp geometric separation between the vertices of $G$ that are in $H$ and those that are not. Consider any drawing $\Gamma_{j}$ with $j \in\{1, \ldots, k\}$ (where $\Gamma_{1}$ is considered before moving $u_{0}$ by $d^{*}$ units to the left) and let $D_{j}$ be the disk centered at $u_{j}$ with radius $d\left(\Gamma_{j}, u_{j-1} u_{j}\right)$. By Properties 1 and 2 of $\Gamma_{j}$, the path $\tau_{u_{j-1} u_{j}}\left(G_{j}\right)$ lies on $\overline{u_{j-1} u_{j}}$ in $\Gamma_{j}$, hence it lies inside $D_{j}$. Further, the edges of $\beta_{u_{j-1} u_{j}}\left(G_{j}\right)$ have slopes in $\left(-\alpha^{\prime}, \alpha^{\prime}\right) \subset\left(-\frac{\alpha}{8}, \frac{\alpha}{8}\right) \subset\left(-\frac{\pi}{32}, \frac{\pi}{32}\right)$; hence $\beta_{u_{j-1} u_{j}}\left(G_{j}\right)$ also lies inside $D_{j}$. By planarity $\Gamma_{j}$ lies entirely inside $D_{j}$. Hence, $u_{j-1}$ is the farthest vertex of $G_{j}$ from $u_{j}$ in $\Gamma_{j}$. This property is true also after the uniform scaling of $\Gamma_{1}, \ldots, \Gamma_{k}$; further, after the scaling, the distance between $u_{j-1}$ and $u_{j}$ is $\frac{\rho}{k}$, by construction. By the triangular inequality, we have that $d\left(\Gamma_{\delta}, v z\right) \leq \sum_{j=i+1}^{k} d\left(\Gamma_{\delta}, u_{j-1} u_{j}\right)+d\left(\Gamma_{\delta}, u_{i} z\right)$. Since $d\left(\Gamma_{\delta}, u_{j-1} u_{j}\right)=\frac{\rho}{k}$ for any $j \in\{2, \ldots, k\}$, and since $d\left(\Gamma_{\delta}, u_{i} z\right) \leq \frac{\rho}{k}$ (this exploits $z \neq u_{0}$ and hence $d\left(\Gamma_{\delta}, u_{i} z\right)=d\left(\Gamma_{i}, u_{i} z\right)$, where $\Gamma_{i}$ is understood as already scaled), we have that $d\left(\Gamma_{\delta}, v z\right) \leq \frac{(k-i+1) \rho}{k} \leq \rho$. Thus $z$ lies inside $D_{\rho}$.

We now prove that Property 6 is satisfied by $\Gamma_{\delta}$. We devote our attention to the proof of the existence of a distance-decreasing path $P_{x y}$ from a vertex $x$ to a vertex $y$ if: (i) $x \in V(H)$ and $y \in V\left(G_{i}\right)$, for some $i \in\{1, \ldots, k\}$; or (ii) $x \in V\left(G_{i}\right)$, for some $i \in\{1, \ldots, k\}$, and $y \in V(H)$. While the rest of the proof that Property 6 is satisfied by $\Gamma_{\delta}$ proceeds similarly to the proof of Lemma 10, cases (i) and (ii) above deal with vertices $x$ and $y$ that are "far apart" in $\Gamma_{\delta}$, a circumstance that does not occur in the proof of Lemma 10.

In case (i) $P_{x y}$ contains a path $P_{x}^{H}$ in $H$ from $x$ to $y_{1}$. Assume that $x \neq u$, as the case $x=u$ is easier to handle. By Property 4 of $\Gamma_{H, \delta}$, there is a path $P_{x}^{H}=\left(x=z_{1}, \ldots, z_{s}=y_{1}\right)$ in $H$ that connects $x$ to $y_{1}$, that does not pass through $u$, and whose edges have slopes in $\left(-\frac{\alpha}{2}, \frac{\alpha}{2}\right)$ in $\Gamma_{H, \delta}$. We prove that, for $h=1, \ldots, s-1, d\left(\Gamma_{\delta}, z_{h} y\right)>d\left(\Gamma_{\delta}, z_{h+1} y\right)$; see Fig. 9 . Since the drawing of $H$ in $\Gamma_{\delta}$ coincides with $\Gamma_{H, \delta}, z_{h} z_{h+1}$ has slope in $\left(-\frac{\alpha}{2}, \frac{\alpha}{2}\right)$ in $\Gamma_{\delta}$. Let $\ell_{h}$ be the directed line through $y_{1}$ directed towards increasing $y$-coordinates and orthogonal to the line through $z_{h}$ and $z_{h+1}$. Denote by $s_{h}$ the slope of $\ell_{h}$. Then $s_{h} \in\left(\frac{\pi-\alpha}{2}, \frac{\pi+\alpha}{2}\right)$.

We prove that $\ell_{h}$ has the disk $D_{\rho}$ to its right. In order to do that, consider the point $p_{T}$ on the half-line with slope $\frac{\pi-\alpha}{2}$ starting at $y_{1}$ and such that $d_{V}\left(\Gamma_{\delta}, y_{1} p_{T}\right)=\rho$. Further, consider 


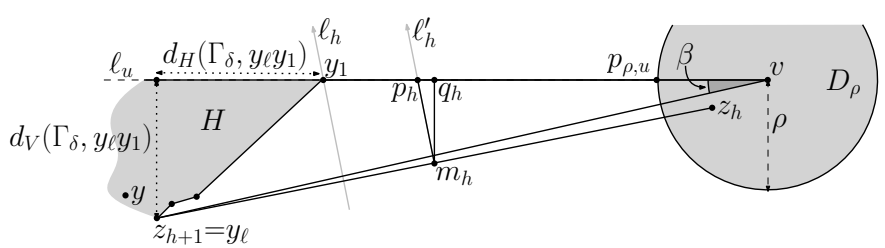

Figure 10 Illustration for the proof that $d\left(\Gamma_{\delta}, z_{h} y\right)>d\left(\Gamma_{\delta}, z_{h+1} y\right)$ if $z_{h+1}=y_{\ell}=u_{0}$.

the point $p_{B}$ on the half-line with slope $\frac{-\pi+\alpha}{2}$ starting at $y_{1}$ and such that $d_{V}\left(\Gamma_{\delta}, y_{1} p_{B}\right)=\rho$. Note that $\overline{p_{T} p_{B}}$ is a vertical straight-line segment with length $2 \rho$. Consider the infinite closed strip $S$ with height $2 \rho$ that is delimited by the horizontal lines through $p_{T}$ and $p_{B}$. Since $D_{\rho}$ has its center on $\ell_{u}$ and has radius $\rho$, it lies inside $S$. The part of $\ell_{h}$ inside $S$ is to the left of $\overline{p_{T} p_{B}}$, given that $s_{h} \in\left(\frac{\pi-\alpha}{2}, \frac{\pi+\alpha}{2}\right)$. Hence, it suffices to show that $p_{\rho, u}$, which is the point of $D_{\rho}$ with smallest $x$-coordinate, lies to the right of $\overline{p_{T} p_{B}}$. We have $d\left(\Gamma_{\delta}, y_{1} p_{\rho, u}\right)=d_{y_{1} v}-\rho$. Further, $d_{H}\left(\Gamma_{\delta}, y_{1} p_{T}\right)=\rho \cdot \tan \left(\frac{\alpha}{2}\right)$. Hence, it suffices to prove $\rho \cdot \tan \left(\frac{\alpha}{2}\right)<d_{y_{1} v}-\rho$, that is $\rho<\frac{d_{y_{1} v}}{1+\tan \left(\frac{\alpha}{2}\right)} ;$ this is true since $\rho<\frac{d_{y_{1} v}}{3}$ and $\tan \left(\frac{\alpha}{2}\right)<1$, given that $0<\alpha<\frac{\pi}{4}$.

The line $\ell_{h}$ has $\Gamma_{H, \delta}$ (and in particular the midpoint of $z_{h} z_{h+1}$ ) to its left; this is because by Property 2 of $\Gamma_{H, \delta}$ every edge in $\beta_{y_{1} u}(H)$ has slope $\pi$, where $s_{h}<\frac{\pi+\alpha}{2}<\pi<\frac{3 \pi-\alpha}{2}<\pi+s_{h}$, and because by Property 3 of $\Gamma_{H, \delta}$ every edge in $\tau_{y_{1} u}(H)$ has slope in $\left(\pi-\frac{\alpha}{2}, \pi+\frac{\alpha}{2}\right)$, where $s_{h}<\frac{\pi+\alpha}{2}<\pi-\frac{\alpha}{2}<\pi+\frac{\alpha}{2}<\frac{3 \pi-\alpha}{2}<\pi+s_{h}$. Let $\ell_{h}^{\prime}$ be the directed line parallel to $\ell_{h}$, passing through the midpoint of $z_{h} z_{h+1}$, and oriented towards increasing $y$-coordinates; $\ell_{h}^{\prime}$ has $\ell_{h}$ to its right, as the midpoint of $z_{h} z_{h+1}$ is to the left of $\ell_{h}$ in $\Gamma_{\delta}$. Thus, $\ell_{h}^{\prime}$ has $D_{\rho}$, and in particular $y$, to its right. Since the half-plane to the right of $\ell_{h}^{\prime}$ is the locus of the points of the plane that are closer to $z_{h+1}$ than to $z_{h}$, it follows that $d\left(\Gamma_{\delta}, z_{h} y\right)>d\left(\Gamma_{\delta}, z_{h+1} y\right)$.

The path $P_{x y}$ also contains the edge $y_{1} v$, which "jumps" from $H$ to $D_{\rho}$. Since $y$ lies in $D_{\rho}$, we have that $d\left(\Gamma_{\delta}, v y\right) \leq \rho \leq \frac{d_{y_{1} v}}{3}$. By the triangular inequality, we have that $d\left(\Gamma_{\delta}, y_{1} y\right)>d\left(\Gamma_{\delta}, y_{1} v\right)-d\left(\Gamma_{\delta}, v y\right) \geq d_{y_{1} v}-\rho \geq \frac{2 d_{y_{1} v}}{3}$. Hence, $d\left(\Gamma_{\delta}, y_{1} y\right)>d\left(\Gamma_{\delta}, v y\right)$. The third sub-path of $P_{x y}$ is a path $P_{v y}$ from $v$ to $y$ in $\bigcup_{l=i}^{k} G_{l}$ that is distance-decreasing in $\Gamma_{\delta}$. The construction of this path proceeds similarly as in the proof of Lemma 10.

In case (ii) we have that $x \in V\left(G_{i}\right)$, for some $i \in\{1, \ldots, k\}$, and $y \in V(H)$. While in case (i) the connection between $H$ and $D_{\rho}$ is done via $y_{1}$, here it is done via $y_{\ell}$. In particular, the first part of $P_{x y}$ consists of edges with slopes in the range $(\pi-\alpha, \pi+\alpha)$ inside $D_{\rho}$. Similarly to case (i), the orthogonal line through the midpoint of each of these edges separates $H$ from $D_{\rho}$; hence traversing the edge decreases the distance to $y$.

We now argue that traversing an edge that "jumps" from $D_{\rho}$ to $H$ decreases the distance to $y$. That is, we show that, for a vertex $z_{h}$ in $D_{\rho}$ incident to an edge $z_{h} z_{h+1}$ with $z_{h+1}=y_{\ell}=u_{0}$, it holds $d\left(\Gamma_{\delta}, z_{h} y\right)>d\left(\Gamma_{\delta}, z_{h+1} y\right)$. Refer to Fig. 10. We exploit again the fact that the line $\ell_{h}$ passing through $y_{1}$ and orthogonal to the line through $z_{h}$ and $z_{h+1}$ has $\Gamma_{H, \delta}$ (and in particular $y$ ) to its left; then consider the directed line $\ell_{h}^{\prime}$ parallel to $\ell_{h}$, oriented towards increasing $y$-coordinates, and passing through the midpoint $m_{h}$ of $z_{h} z_{h+1}$. Since the half-plane to the left of $\ell_{h}^{\prime}$ is the locus of the points of the plane that are closer to $z_{h+1}$ than to $z_{h}$, it suffices to show that the intersection point $p_{h}$ of $\ell_{h}^{\prime}$ and $\ell_{u}$ lies to the right of $y_{1}$ on $\ell_{u}$; in fact, this implies that $\ell_{h}^{\prime}$ has $\ell_{h}$ (and hence $y$ ) to its left.

Since $z_{h}$ lies inside $D_{\rho}$, we have $x\left(z_{h}\right) \geq x\left(p_{\rho, u}\right)=x\left(y_{1}\right)+d_{y_{1} v}-\rho$. Moreover, $x\left(y_{1}\right)=$ $x\left(y_{\ell}\right)+d_{H}\left(\Gamma_{\delta}, y_{\ell} y_{1}\right)$. Thus, we have $x\left(m_{h}\right)=\frac{x\left(y_{\ell}\right)+x\left(z_{h}\right)}{2} \geq \frac{x(y \ell)+\left(x\left(y_{\ell}\right)+d_{H}\left(\Gamma_{\delta}, y_{\ell} y_{1}\right)+d_{y_{1} v}-\rho\right)}{2}=$ $x\left(y_{\ell}\right)+\frac{d_{H}\left(\Gamma_{\delta}, y_{\ell} y_{1}\right)+d_{y_{1} v}-\rho}{2}$. Translate the Cartesian axes so that $x\left(y_{\ell}\right)=0$. Thus, $x\left(m_{h}\right)=$ $\frac{d_{H}\left(\Gamma_{\delta}, y_{\ell} y_{1}\right)+d_{y_{1} v}-\rho}{2}$. By Lemma $7, y_{\ell}$ is an internal vertex of $\beta_{u v}(G)$, hence $y_{\ell}$ lies below $\ell_{u}$. 
Since $\rho<Y$ and $z_{h}$ lies in $D_{\rho}$, the $y$-coordinate of $y_{\ell}$ is smaller than the one of $z_{h}$. Hence, the slope of $z_{h} z_{h+1}$ is larger than $\pi$. Further, $z_{h}$ and hence $m_{h}$ lie on or below $h_{\beta}$, thus the slope of $z_{h} z_{h+1}$ is at most $\pi+\beta$ and the slope $s_{h}$ of $\ell_{h}^{\prime}$ is in the interval $\left(\frac{\pi}{2}, \frac{\pi}{2}+\beta\right)$.

We now derive a lower bound for the $x$-coordinate of $p_{h}$. Let $q_{h}$ be the projection of $m_{h}$ on $\ell_{u}$. Consider the triangle $\Delta m_{h} p_{h} q_{h}$. Since the $y$-coordinate of $y_{\ell}$ is smaller than the one of $z_{h}$, it is also smaller than the one of $m_{h}$. Thus, $d\left(\Gamma_{\delta}, m_{h} q_{h}\right) \leq d_{V}\left(\Gamma_{\delta}, y_{\ell} y_{1}\right)$. Since $s_{h} \in\left(\frac{\pi}{2}, \frac{\pi}{2}+\beta\right)$, the angle $\measuredangle p_{h} m_{h} q_{h}$ is at most $\beta$. Hence, $d\left(\Gamma_{\delta}, p_{h} q_{h}\right) \leq d_{V}\left(\Gamma_{\delta}, y_{\ell} y_{1}\right) \cdot \tan (\beta)$. Thus, $x\left(p_{h}\right)=x\left(m_{h}\right)-d\left(\Gamma_{\delta}, p_{h} q_{h}\right) \geq \frac{d_{H}\left(\Gamma_{\delta}, y_{\ell} y_{1}\right)+d_{y_{1} v}-\rho}{2}-d_{V}\left(\Gamma_{\delta}, y_{\ell} y_{1}\right) \cdot \tan (\beta)$. It remains to prove that this quantity is larger than $d_{H}\left(\Gamma_{\delta}, y_{\ell} y_{1}\right)$, which is the $x$-coordinate of $y_{1}$.

Since $\beta<\frac{\alpha}{4}<\frac{\pi}{16}$, we have $\tan (\beta) \leq 1$, hence $\frac{d_{H}\left(\Gamma_{\delta}, y_{\ell} y_{1}\right)+d_{y_{1} v}-\rho}{2}-d_{V}\left(\Gamma_{\delta}, y_{\ell} y_{1}\right) \cdot \tan (\beta) \geq$ $\frac{d_{H}\left(\Gamma_{\delta}, y_{\ell} y_{1}\right)+d_{y_{1} v}-\rho}{2}-d_{V}\left(\Gamma_{\delta}, y_{\ell} y_{1}\right)$. We want to establish $\frac{d_{H}\left(\Gamma_{\delta}, y_{\ell} y_{1}\right)+d_{y_{1} v}-\rho}{2}-d_{V}\left(\Gamma_{\delta}, y_{\ell} y_{1}\right)>$ $d_{H}\left(\Gamma_{\delta}, y_{\ell} y_{1}\right)$, that is, $d_{y_{1} v}>2 d_{V}\left(\Gamma_{\delta}, y_{\ell} y_{1}\right)+d_{H}\left(\Gamma_{\delta}, y_{\ell} y_{1}\right)+\rho$. Since $\rho \leq \frac{d_{y_{1} v}}{3}$, we need to prove that $d_{y_{1} v}>\frac{6 d_{V}\left(\Gamma_{\delta}, y_{\ell} y_{1}\right)+3 d_{H}\left(\Gamma_{\delta}, y_{\ell} y_{1}\right)}{2}$.

We now express $d_{y_{1} v}$ as a function of $\beta$. This is done by looking at the triangle whose vertices are $y_{\ell}, v$, and the projection of $y_{\ell}$ on $\ell_{u}$. Since the angle of this triangle at $v$ is $\beta$, we get $d_{y_{1} v}=\frac{d_{V}\left(\Gamma_{\delta}, y_{\ell} y_{1}\right)}{\tan (\beta)}-d_{H}\left(\Gamma_{\delta}, y_{\ell} y_{1}\right)$. Substituting this into the previous inequality, we need to have $\frac{d_{V}\left(\Gamma_{\delta}, y_{\ell} y_{1}\right)}{\tan (\beta)}-d_{H}\left(\Gamma_{\delta}, y_{\ell} y_{1}\right)>\frac{6 d_{V}\left(\Gamma_{\delta}, y_{\ell} y_{1}\right)+3 d_{H}\left(\Gamma_{\delta}, y_{\ell} y_{1}\right)}{2}$, hence $\tan (\beta)<$ $\frac{2 d_{V}\left(\Gamma_{\delta}, y_{\ell} y_{1}\right)}{6 d_{V}\left(\Gamma_{\delta}, y_{\ell} y_{1}\right)+5 d_{H}\left(\Gamma_{\delta}, y_{\ell} y_{1}\right)}$, which is true since $\beta<\arctan \left(\frac{d_{V}\left(\Gamma_{H}, y_{\ell} y_{1}\right)}{3 d_{V}\left(\Gamma_{H}, y_{\ell} y_{1}\right)+3 d_{H}\left(\Gamma_{H}, y_{\ell} y_{1}\right)}\right)$. This concludes the proof that $d\left(\Gamma_{\delta}, z_{h} y\right)>d\left(\Gamma_{\delta}, z_{h+1} y\right)$.

The path $P_{x y}$ continues with a path $P_{y_{\ell} y}$ from $y_{\ell}$ to $y$ in $H$ that is distance-decreasing in $\Gamma_{H, \delta}$ (and hence in $\Gamma_{\delta}$, since the drawing of $H$ in $\Gamma_{\delta}$ coincides with $\Gamma_{H, \delta}$ ). This concludes the proof of the lemma.

Given a strong circuit graph $(G, u, v)$ such that $G$ is not a single edge, we are in Case A or Case B depending on whether the edge $u v$ exists or not, respectively. Thus, Lemmata 9-11 prove Theorem 8 . We show how to use Theorem 8 in order to prove Theorem 3 . Consider any 3-connected planar graph $G$ and associate any plane embedding to it; let $u$ and $v$ be two consecutive vertices in the clockwise order of the vertices along the outer face of $G$. We have that $(G, u, v)$ is a strong circuit graph. Indeed: (a) by assumption $G$ is 2-connected in fact 3-connected - and associated with a plane embedding; (b) by construction $u$ and $v$ are two distinct external vertices of $G$; (c) edge $u v$ exists and coincides with $\tau_{u v}(G)$, given that $v$ immediately follows $u$ in the clockwise order of the vertices along the outer face of $G$; and (d) $G$ does not have any 2 -cut, as it is 3 -connected. Thus, Theorem 8 can be applied in order to construct a planar greedy drawing of $G$. This concludes the proof of Theorem 3 .

\section{Conclusions}

In this paper we have shown how to construct planar greedy drawings of 3-connected planar graphs. It is tempting to try to use the graph decomposition we employed in this paper for proving that 3-connected planar graphs admit convex greedy drawings. However, despite some efforts in this direction, we have not been able to modify the statement of Theorem 8 in order to guarantee the desired convexities of the angles in the drawings. Thus, proving or disproving the convex greedy embedding conjecture remains an elusive goal.

\section{References}

1 S. Alamdari, T. M. Chan, E. Grant, A. Lubiw, and V. Pathak. Self-approaching graphs. In Didimo and Patrignani, editors, GD, volume 7704 of $L N C S$, pages 260-271, 2012. 
2 P. Angelini, G. Di Battista, and F. Frati. Succinct greedy drawings do not always exist. Networks, 59(3):267-274, 2012.

3 P. Angelini, E. Colasante, G. Di Battista, F. Frati, and M. Patrignani. Monotone drawings of graphs. J. Graph Algorithms Appl., 16(1):5-35, 2012.

4 P. Angelini, F. Frati, and L. Grilli. An algorithm to construct greedy drawings of triangulations. J. Graph Algorithms Appl., 14(1):19-51, 2010.

5 D. Barnette. Trees in polyhedral graphs. Canadian J. Math., 18:731-736, 1966.

6 P. Bose, P. Morin, I. Stojmenović, and J. Urrutia. Routing with guaranteed delivery in ad hoc wireless networks. Wireless Networks, 7(6):609-616, 2001.

7 G. Chen and X. Yu. Long cycles in 3-connected graphs. J. Comb. Theory, Ser. B, 86(1):8099, 2002 .

8 G. Da Lozzo, A. D'Angelo, and F. Frati. On planar greedy drawings of 3-connected planar graphs. CoRR, 2016. URL: http://arxiv.org/abs/1612.09277.

9 G. Da Lozzo, V. Dujmović, F. Frati, T. Mchedlidze, and V. Roselli. Drawing planar graphs with many collinear vertices. In $\mathrm{Hu}$ and Nöllenburg, editors, GD, volume 9801 of $L N C S$, pages $152-165,2016$.

10 H. R. Dehkordi, F. Frati, and J. Gudmundsson. Increasing-chord graphs on point sets. J. Graph Algorithms Appl., 19(2):761-778, 2015.

11 R. Dhandapani. Greedy drawings of triangulations. Discr. Comp. Geom., 43(2):375-392, 2010 .

12 G. Di Battista and R. Tamassia. Algorithms for plane representations of acyclic digraphs. Theor. Comput. Sci., 61:175-198, 1988.

13 D. Eppstein and M. T. Goodrich. Succinct greedy geometric routing using hyperbolic geometry. IEEE Trans. Computers, 60(11):1571-1580, 2011.

14 S. Felsner, A. Igamberdiev, P. Kindermann, B. Klemz, T. Mchedlidze, and M. Scheucher. Strongly monotone drawings of planar graphs. In Fekete and Lubiw, editors, SoCG, volume 51 of $L I P I c s$, pages 37:1-37:15, 2016.

15 H. Frey, S. Rührup, and I. Stojmenović. Routing in wireless sensor networks. In Misra, Woungang, and Misra, editors, Guide to Wireless Sensor Networks, Computer Communications and Networks, chapter 4, pages 81-111. Springer, 2009.

16 M. T. Goodrich and D. Strash. Succinct greedy geometric routing in the Euclidean plane. In Dong, Du, and Ibarra, editors, ISAAC, volume 5878 of $L N C S$, pages 781-791, 2009.

$17 \mathrm{X}$. He and H. Zhang. On succinct greedy drawings of plane triangulations and 3-connected plane graphs. Algorithmica, 68(2):531-544, 2014.

18 P. Kindermann, A. Schulz, J. Spoerhase, and A. Wolff. On monotone drawings of trees. In Duncan and Symvonis, editors, GD, volume 8871 of $L N C S$, pages 488-500, 2014.

19 E. Kranakis, H. Singh, and J. Urrutia. Compass routing on geometric networks. In $C C C G$, 1999. URL: http://www.cccg.ca/proceedings/1999/c46.pdf.

20 F. Kuhn, R. Wattenhofer, and A. Zollinger. An algorithmic approach to geographic routing in ad hoc and sensor networks. IEEE/ACM Trans. Netw., 16(1):51-62, 2008.

21 T. Leighton and A. Moitra. Some results on greedy embeddings in metric spaces. Discr. Comp. Geom., 44(3):686-705, 2010.

22 M. Nöllenburg and R. Prutkin. Euclidean greedy drawings of trees. In Bodlaender and Italiano, editors, ESA, volume 8125 of $L N C S$, pages 767-778, 2013.

23 M. Nöllenburg, R. Prutkin, and I. Rutter. On self-approaching and increasing-chord drawings of 3-connected planar graphs. J. Comp. Geom., 7(1):47-69, 2016.

24 C. H. Papadimitriou and D. Ratajczak. On a conjecture related to geometric routing. In Nikoletseas and Rolim, editors, ALGOSENSORS, volume 3121 of LNCS, pages 9-17, 2004

25 C. H. Papadimitriou and D. Ratajczak. On a conjecture related to geometric routing. Theor. Comput. Sci., 344(1):3-14, 2005. 
26 A. Rao, C. H. Papadimitriou, S. Shenker, and I. Stoica. Geographic routing without location information. In Johnson, Joseph, and Vaidya, editors, MOBICOM, pages 96-108, 2003.

27 C. Siva Ram Murthy and B.S. Manoj. Ad Hoc Wireless Networks: Architectures and Protocols. Prentice Hall, 2004.

28 C. K. Toh. Ad Hoc Mobile Wireless Networks: Protocols and Systems. Prentice Hall, 2002. 